\title{
A thirteen-year analysis of Plasmodium falciparum populations reveals high conservation of the mutant pfcrt haplotype despite the withdrawal of chloroquine from national treatment guidelines in Gabon
}

\author{
Matthias Frank ${ }^{1,2^{*}+}$, Nicola Lehners ${ }^{1 \dagger}$, Pembe I Mayengue ${ }^{1,5}$, Julian Gabor ${ }^{1,2}$, Matthias Dal-Bianco ${ }^{1,2}$, \\ David U Kombila1,2, Ghyslain Mombo Ngoma ${ }^{1,2,6}$, Christian Supan ${ }^{1,2}$, Bertrand Lell ${ }^{1,2}$, Francine Ntoumi ${ }^{1,2,5}$, \\ Martin P Grobusch ${ }^{1,2,4}$, Klaus Dietz ${ }^{3}$ and Peter G Kremsner ${ }^{1,2}$
}

\begin{abstract}
Background: Chloroquine resistance (CR) decreased after the removal of chloroquine from national treatment guidelines in Malawi, Kenia and Tanzania. In this investigation the prevalence of the chloroquine resistance (CQR) conferring mutant pfcrt allele and its associated chromosomal haplotype were determined before and after the change in Gabonese national treatment guidelines from chloroquine (CQ) to artesunate plus amodiaquine (AQ) in 2003.

Methods: The prevalence of the wild type pfcrt allele was assessed in 144 isolates from the years 2005 - 07 by PCR fragment restriction digest and direct sequencing. For haplotype analysis of the chromosomal regions flanking the pfcrt locus, microsatellite analysis was done on a total of 145 isolates obtained in 1995/96 (43 isolates), 2002 (47 isolates) and 2005 - 07 (55 isolates).
\end{abstract}

Results: The prevalence of the mutant pfcrt allele decreased from 100\% in the years 1995/96 and 2002 to $97 \%$ in 2005 - 07. Haplotype analysis showed that in 1995/96 79\% of the isolates carried the same microsatellite alleles in a chromosomal fragment spanning $39 \mathrm{~kb}$ surrounding the pfcrt locus. In 2002 and 2005 - 07 the prevalence of this haplotype was $62 \%$ and 58\%, respectively. Pfcrt haplotype analysis showed that all wild type alleles were CVMNK.

Conclusion: Four years after the withdrawal of CQ from national treatment guidelines the prevalence of the mutant pfcrt allele remains at $97 \%$. The data suggest that the combination of artesunate plus AQ may result in continued selection for the mutant pfcrt haplotype even after discontinuance of CQ usage.

Keywords: chloroquine resistance, chloroquine sensitivity, microsatellite analysis, haplotype analysis, artesunate plus amodiaquine

\section{Background}

The evolution of $C Q R$ has been a major obstacle to global public health [1]. CQR reached Africa in the late seventies and reached a high prevalence across the continent over the following decade [2]. Malawi was the

\footnotetext{
* Correspondence: Matthias.frank@uni-tuebingen.de

† Contributed equally

'Institute of Tropical Medicine, University of Tübingen, Wilhelmstr.27, 72074

Tübingen, Germany

Full list of author information is available at the end of the article
}

first African country to discontinue the use of CQ in 1993 [3]. Interestingly, the prevalence of the resistant pfcrt allele $76 \mathrm{~T}$ decreased drastically from $85 \%$ to $13 \%$ within the following eight years $[3,4]$. In 2005, a clinical trial showed a $100 \%$ efficacy of CQ for treatment of uncomplicated Plasmodium falciparum malaria in children [5]. Recently, Kenya and Tanzania have also reported a decline in CQR after replacement of CQ by sulphadoxine-pyrimethamine (SP), however, this decline occurred at a much slower rate than in Malawi [6,7]. 
The genetic cause of CQR was found to be a single point mutation changing the amino acid lysine $(\mathrm{K})$ to threonine (T) at position 76 in the gene coding for the $P$. falciparum CQ resistance transporter (pfcrt) [8-10]. This mutation arose only in a small number of founder locations, from where the resistant parasites spread worldwide. As a consequence, the variability of the chromosomal regions flanking the pfcrt gene on chromosome 7 is strongly reduced in resistant parasites, a phenomenon referred to as selective sweep [11]. The chromosomal haplotype - a characterization of this part of the chromosome, assessed either by SNP or microsatellite analysis - is, therefore, highly conserved in resistant parasites and specific for the respective founder locations. In contrast, sensitive parasites generally have very diverse haplotypes $[11,12]$.

In Gabon, CQ use was officially discontinued in 2003 and replaced by the combination of artesunate and AQ. Before the change in national treatment guidelines the prevalence of the mutant pfcrt allele $76 \mathrm{~T}$ was repeatedly measured to be $100 \%$ [13-15]. The goal of this study is to investigate if this change was associated with a return of the wild type pfcrt allele - as has been observed in other African countries - and if it affected genetic diversity in the surrounding chromosomal areas.

\section{Methods}

\section{Study site and population}

All samples were taken from clinical studies conducted at the Albert-Schweitzer-Hospital in Lambaréné, Gabon. Malaria in Lambaréné is hyperendemic with stable transmission throughout the year, the predominant species is P. falciparum [16]. Patients had been recruited in Lambaréné and surroundings (radius approx. $60 \mathrm{~km}$ ). Informed consent had been obtained by all patients or their guardians and all studies had been approved by the Ethics Committee of the International Foundation of the Albert Schweitzer Hospital. For the 1995/96 cohort, samples from the $1 / 95 \mathrm{C}$ study were analysed [17]. The 2002 cohort consisted of samples from the 2002 case control study [15,18]. For the years 2005 - 07 samples were obtained from the Antigenic Diversity Study $[19,20]$, the Ferroquine Tolerance Trial [21], the IPTi Study [22,23] and the SP Efficacy Trial [24].

\section{DNA extraction}

Parasite DNA was extracted either from whole blood samples or from dried filter blood spots using the QIAamp $^{\circledR}$ DNA Mini Kit or DNA Blood Mini Kit (Qiagen, Hilden, Germany) according to the manufacturer's protocol. Extracted DNA was stored at $-20^{\circ} \mathrm{C}$.

\section{Polymerase chain reaction and sequencing}

Polymerase chain reaction (PCR) was carried out using $2.5 \mathrm{mM} \mathrm{MgCl}_{2}, 0.2 \mathrm{mM}$ denucleoside triphosphates, 0.5 $\mu \mathrm{M}$ forward and reverse primer, 1.5 $\mathrm{U}$ Taq polymerase and $5 \mu \mathrm{l}$ DNA in a total volume of $50 \mu \mathrm{l}$. In case of nested PCRs $5 \mu \mathrm{l}$ PCR product of the first amplification step was used as template in the second step. PCR reagents were obtained from Qiagen, primers were ordered from Operon (Cologne, Germany). Reactions were run in a Biometra Uno II Thermal Cycler (Biometra, Goettingen, Germany), a MyCycler Thermal Cycler (Bio-Rad, Munich, Germany) and a MJ Research PTC200 (GMI, Ramsey, USA). PCR products were stained with SYBR Green (Biozym, Hess. Oldendorf, Germany) and run on a $1.5 \%$ agarose gel. PCR products submitted to sequencing were cut out of the gel, extracted and purified using the NucleoSpin Extract II Kit (MacheryNagel, Dueren, Germany) according to the manufacturer's protocol. This was followed by a sequencing PCR using 1/10 BigDye Terminator (Applied Biosystems, Warrington, UK) and approx. $1.5 \mathrm{ng}$ DNA/100 bp. 25 cycles were run consisting of melting at $94^{\circ} \mathrm{C}$ for $10 \mathrm{sec}$, annealing at $50^{\circ} \mathrm{C}$ for $5 \mathrm{sec}$ and elongation at $60^{\circ} \mathrm{C}$ for 4 min. Obtained PCR products were purified using Sephadex 96-well plates (GE Healthcare, Little Chalfont, UK) and sequenced by a Abi Prism 3000 Gene Analyzer (Applied Biosystems). Sequences were displayed with the BioEdit software (Ibis Biosciences, Carlsbad, USA).

\section{Detection of pfcrt mutations by restriction digest and sequencing}

CQR of isolates was determined by detection of the mutant or wild type allele of the pfcrt gene at position 76. The CQR conferring mutation K76T as well as seven other resistance associated mutations (M74I, N75E, A220S, Q271E, N326S, I356T, R371I) were detected using a method consisting of a nested PCR followed by mutation specific restriction digestion as described by Djimde et al [25]. The mutations M74I and N75E were directly detected via PCR product sequencing.

\section{Haplotype analysis}

The chromosomal haplotype was defined as the specific combination of the alleles of the following six microsatellite markers: PE14D (distance from the pfcrt gene -45 kb), B5M77 (-18 kb), 3E7 (-11 kb), 9B12 (+2 kb), 7A11 $(+21 \mathrm{~kb})$ and PE14F (+57 kb). Additionally, the microsatellite B5M47 (-0 kb) was analysed in several isolates of the years 2005 - 07 but not taken into account for the haplotype analysis. The position of the individual microsatellites relative to $p f c r t$ is calculated based on their coordinates on Chromosome 7 of the 3D7 genome strain (http://www.plasmodb.org). These differ somewhat from the originally reported distances by Wootton et al [11]. Alleles were determined as differences in repeat length. The repeat sequence motifs of the 
different microsatellites were as follows: PE14D (TAC)/ (TAA), B5M77 (TA), 3E7 (TA)/(T)/(TA), 9B12 (TA), 7A11 (TTATA), PE14F (TAA). In case of multiple infections only samples with a clear dominance of one microsatellite allele in the sequence ferrogram were included for further analysis [12].

Microsatellite markers were amplified via PCR and sequenced. Primers and conditions were adapted from $\mathrm{Su}$ et al [26] but resulted in suboptimal amplification in field isolates. Therefore conditions had to be modified for all microsatellites and in most cases a nested PCR approach had to be implemented (see Additional file 1). The strains 3D7, Dd2 and FCR3 were used as positive PCR controls. The consistency of primers and conditions was verified by sequencing of the PCR amplification products of the control strains and comparing the obtained microsatellite sequences with the known sequences deposited in the NCBI database, which always showed a perfect match of the repeat lengths.

Chromosomal haplotype studies were done on 171 resistant samples from the years 1995 - 2007. Successful sequencing of the full set of microsatellites was achieved in 132 samples. In 145 samples at least the inner four microsatellites (B5M77, 3E7, 9B12, 7A11) covering a chromosomal fragment of approximately $40 \mathrm{~kb}$ could be sequenced. These samples consisted of 43 samples from the 1995/96 cohort, 47 samples of the 2002 cohort and 55 samples of the 2005 - 7 cohort. All sequences were submitted to Genbank. Individual samples can be identified through their study specific label, consisting of a study specific combination of letters and an isolate specific running number, as indicated below: 1/95C study ( $\mathrm{M}$ or S, followed by a number, i.e. M1), 2002 case control study (N followed by a number, i.e. N1), Antigenic Diversity Study (three individual letters, i.e. MOA), the Ferroquine Tolerance Trial (TDU or FED or TDR, followed by a number, i.e. TDU1), IPTi Study (IP followed by a number, i.e. IP1), SP Efficacy Trial (SP followed by a number, i.e. SP1). Genbank accession numbers are: 3E7 sequences: HQ417163 -HQ417327, 7A11 sequences: HQ417328 - HQ417497, 9B12 sequences: HQ417498HQ417655, B5M77 sequences: HQ417656 - HQ417824, PE14D sequences: HQ417825 - HQ417986, PE14F sequences: HQ417987-HQ418152.

\section{msp2 genotyping}

50 isolates from all four studies of the years 2005- 07 were further characterized by sequencing of the central region of the merozoite surface protein 2 ( $m s p 2$ ) gene. Primers and conditions for an allele specific nested PCR were as described by [27]. MSP2 $1^{\text {st }}$ step: forward primer F1 ATGAAGGTAATTAAAACATTGTCTATTATA and reverse primer F2 ATATGGCAAAAGAT AAAACAAGTGTTGCTG, $94^{\circ} \mathrm{C}$ for $5 \mathrm{~min}$, followed by
30 cycles of $94^{\circ} \mathrm{C}$ for $10 \mathrm{sec}, 57^{\circ} \mathrm{C}$ for $30 \mathrm{sec}, 72^{\circ} \mathrm{C}$ for $30 \mathrm{sec}$, and a final extension step of $72^{\circ} \mathrm{C}$ for $2 \mathrm{~min}$. MSP2 $2^{\text {nd }}$ step FC allele: forward primer FC27 F2 GCAAATGAAGGTTCTAATACTAATAG and reverse primer FC27 R2 GCTTTGGGTCCTTCTTCAGTTGATTC, MSP2 $2^{\text {nd }}$ step 3D7 allele: forward primer 3D7 F2 GCAGAAAGTAAGCCTTCTACTGGTGCT and reverse primer 3D7 R2 GATTTGTTTCGGCATTATTATGA. PCR was carried out using the following conditions $94^{\circ} \mathrm{C}$ for $5 \mathrm{~min}$, followed by 40 cycles of $94^{\circ} \mathrm{C}$ for $10 \mathrm{sec}, 57^{\circ} \mathrm{C}$ for $30 \mathrm{sec}, 72^{\circ} \mathrm{C}$ for $30 \mathrm{sec}$, and a final extension step of $72^{\circ} \mathrm{C}$ for $2 \mathrm{~min}$.

\section{Statistical analysis}

Conservation of any marker or haplotype was defined as the prevalence of the dominant allele. Development of the degree of conservation over the years was analysed by logistic regression. A chi-square test was applied for assessment of significance. Allele diversity was described by the expected heterozygosity $H_{e}$ using the formula: $H_{e}$ $=[n /(n-1)]\left[1-\Sigma p_{i}^{2}\right]$ with $n$ being the number of isolates and $p_{i}$ the frequency of the $i$ th allele [28]. Development of $H_{e}$ over the years was analysed by linear regression, an $\mathrm{F}$ test was applied for assessment of significance. In all applied tests $p$-values $<0.05$ were regarded as significant.

\section{Results}

Prevalence of the mutant and wild type pfcrt allele before and after the withdrawal of chloroquine

In order to display the prevalence of the mutant pfcrt allele after 2003, 144 samples from the years $2005-07$ were screened for the CQR conferring mutant 76T pfcrt allele. The restriction digest revealed only one sample with a wild type $76 \mathrm{~K}$ pfcrt allele, thus a genetically sensitive isolate, in 90 samples from the years 2005/06 (1\%) and three isolates with the wild type $76 \mathrm{~K} p f c r t$ allele in 54 samples from 2007 (6\%) (of the wild type isolates three were monoinfections and one was a mixed infection). Overall the prevalence of the mutant $76 \mathrm{~T}$ pfcrt allele remained at $97 \%$ after the withdrawal of chloroquine from national treatment guidelines. Previous analysis of 151 samples from the years 1995/96 and 2002 had shown a prevalence of $100 \%$ for the mutant $76 \mathrm{~T}$ allele [13-15]. For this investigation an additional 64 samples of these studies were analysed. The mutant $76 \mathrm{~T}$ pfcrt allele was detected in all samples. Thus, in total all 215 analysed samples from the years 1995/96 and 2002 carried the mutant $76 \mathrm{~T}$ pfcrt allele, confirming the very high prevalence of the mutant $p f c r t$ allele in this area prior to the change in national treatment guidelines (see Table 1).

Pfcrt haplotype analysis was conducted in the three wild type isolates that were monoinfections and 48 
Table 1 Prevalence of the mutant and wild type pfort allele before and after the withdrawal of chloroquine from national treatment guidelines (2003)

\begin{tabular}{lcc}
\hline & $\mathbf{1 9 9 5 - 2 0 0 2}$ & $\mathbf{2 0 0 5 - 0 7}$ \\
\hline no. of samples & 215 & 144 \\
\hline no. with mutant & $215(100 \%)$ & $141(97 \%)$ \\
\hline no. with wild type & $0(0 \%)$ & $4(3 \%)$ \\
\hline
\end{tabular}

Mutant: pfcrt $76 \mathrm{~T}$

Wild type: pfcrt $76 \mathrm{~K}$

randomly picked mutant isolates from the years 2005 7. Sequencing of the three monoinfections showed that all carried the original wild type pfcrt haplotype CVMNK at the codons 72-76 and AQNIR at the codons $220,271,326,356,371$. The reappeared wild type $p f c r t$ allele thus did not result from a simple back mutation from $\mathrm{T}$ to $\mathrm{K}$ at codon 76 . Sequencing of 48 randomly picked mutant isolates from the years 2005-7 showed that they uniformly carried the CVIET haplotype at the codons 72-76.

\section{Chromosome 7 haplotype characterization of mutant and wild type isolates}

To assess the effect of chloroquine pressure on the chromosomal haplotype, microsatellite allele analysis was initally performed on isolates from 1995/6. For characterization of the chromosome fragment surrounding the pfcrt gene six microsatellite markers were sequenced (PE14D, B5M77, 3E7, 9B12, 7A11, PE14F) covering a chromosomal fragment of approximately 100 $\mathrm{kb}$ (see Figure 1). Analysis of the obtained haplotypes revealed the presence of two most prevalent haplotypes in $1995 / 96$. One haplotype was identical to the haplotype of the resistant lab strain FCR3 [11] (assumed to represent the dominant resistant haplotype in Africa and differing from Dd2 at the microsatellite 7A11) at all six analysed microsatellites and was found in $21 \%$ of the samples. The other haplotype, termed "Lamba", showed a different allele at the most centromeric marker PE14F (see Figure 1) and was prevalent in $37 \%$. Together we defined these two haplotypes as the dominant local resistant haplotypes.

To assess whether the newly appeared pfcrt sensitive isolates from the years 2005-7 represented immigrated strains or local formerly resistant strains that had reacquired the wild type $p f c r t$ allele, haplotype analysis of the wild type isolates was performed. Haplotype analysis was possible in the three monoinfections (samples SP15, SP21, TDU28) of the four wild type isolates. The isolates SP21 and TDU28 carried haplotypes with no resemblance to the dominant local resistant haplotypes. In contrast, the haplotype of isolate SP15 was identical to the dominant local resistant haplotypes at the most telomeric microsatellites PE14D and B5M77 and only started to diverge at microsatellite $3 E 7$, approximately $11 \mathrm{~kb}$ upstream of the pfcrt locus (see Figure 1). This suggested that SP15 might have resulted from a backcross of a wild type pfcrt allele into a local resistant isolate.

\section{Prevalence of the dominant local resistant resistant haplotypes before and after the withdrawal of chloroquine}

The prevalence of the dominant local resistant haplotypes was assessed in 1995/96 and 2002, before the withdrawal of chloroquine from national treatment guidelines, as well as in 2005-7. The dominant local resistant haplotypes maintained a high prevalence at all

\begin{tabular}{|c|c|c|c|c|c|c|c|c|c|}
\hline & $\begin{array}{l}\mathrm{kb} \\
\text { telomere }\end{array}$ & $\begin{array}{c}-45 \\
\text { PE14D } \\
\end{array}$ & $\begin{array}{c}-18 \\
\text { B5M77 } \\
\end{array}$ & $\begin{array}{c}-11 \\
3 E 7 \\
\end{array}$ & $\begin{array}{c}0 \\
\text { pfort }_{72-76} \\
\end{array}$ & $\begin{array}{c}+2 \\
9 \mathrm{~B} 12 \\
\end{array}$ & $\begin{array}{r}+21 \\
7 A 11 \\
\end{array}$ & $\begin{array}{c}+57 \\
\text { PE14F } \\
\end{array}$ & centromere \\
\hline CQR & FCR3 & $8 / 10$ & 11 & $12 / 7 / 12$ & CVIET & 13 & 8 & 11 & \\
\hline CQR & Lamba & $8 / 10$ & 11 & $12 / 7 / 12$ & CVIET & 13 & 8 & 15 & \\
\hline cQs & SP15 & $8 / 10$ & 11 & $17 / 5 / 10$ & CVMNK & 8 & 5 & 10 & \\
\hline cQs & SP21 & $6 / 10$ & 19 & $6 / 7 / 15$ & CVMNK & & 5 & 12 & \\
\hline cQs & TDU28 & $8 / 16$ & 10 & $12 / 5 / 10$ & CVMNK & 8 & 6 & 13 & \\
\hline
\end{tabular}

Figure 1 Haplotype analysis of resistant and sensitive parasites. The two most frequent local resistant haplotypes from the year $1995 / 6$ are displayed. One of the haplotypes is identical to the FCR3 haplotype and represented 21\% of all haplotypes analysed in 1995/6. The other haplotype (denoted "Lamba") represented 37\% of all haplotypes in 1995/6. The haplotypes of the three sensitive monoinfections SP15, SP21 and TDU28 from the years $2005-7$ are represented in the lower part of the figure. Alleles identical to the local resistant haplotypes are shaded in light grey, differing alleles in dark grey. Samples SP21 and TDU28 carry completely different chromosome 7 haplotypes, suggesting that they represent reintroduced sensitive parasites. In contrast SP15 carries the same alleles as the local resistant haplotypes at the microsatellite PE14D and B5M77 and subsequently starts to diverge. This suggests a backcross of the sensitive pfcrt allele. Sequencing of the marker $9 \mathrm{~B} 12$ in the sensitive isolate SP21 was unsuccessful. Haplotypes are represented by indicating the number of repeats at each microsatellite. The pfcrt haplotype at codons $72-76$ is also displayed. 
times although a decrease from 58\% prevalence in 1995/ 96 to $31 \%$ in 2002 and $33 \%$ in $2005-07$ ( $p=0.01$, see Table 2) was noticeable. However, it is striking that the observed development towards lesser conservation took place entirely between 1995/96 and 2002 - thus before the withdrawal of CQ - while no significant change in conservation could be observed between 2002 and 2005 - 07. Besides the dominant local resistant resistant haplotypes, multiple other individual haplotypes were identified. Most of these haplotypes, however, were found only once in the examined population. The total number of unique haplotypes present in the parasite population increased from 18 (including the two dominant haplotypes) in 1995/96 to 28 in 2002 and stayed at 28 in $2005-07$.

To assess genetic change in the chromosomal areas immediately adjacent to the pfcrt locus a local "inner" haplotype was defined as the chromosomal fragment defined by the four microsatellites B5M77, 3E7, 9B12, 7A11, covering a region of approximately $40 \mathrm{~kb}$ around the pfcrt locus. This revealed that in 1995/96 79\% of the analysed isolates carried one identical haplotype in this chromosomal region. In analogy to the six-microsatellite-haplotype, the prevalence of this dominant local "inner" haplotype remained relatively high throughout the years, but showed a statistically significant decrease to $62 \%$ in 2002 and $58 \%$ in $2005-07$ ( $p=0.03$, see Table 3). Again, the decrease in conservation was most pronounced between $1995 / 6$ and 2002, before the change in national treatment guidelines.

To evaluate the propensity for genetic change at the individual microsatellite markers, the change in conservation from 1995/6 to 2005 - 07 was evaluated. This revealed a significant decrease in conservation at the two most centromeric microsatellites 7A11 $(p=0.04)$ and PE14F $(p=0.02)$ while the other microsatellite markers remained conserved.

In order to assess the degree of genetic diversity at the individual microsatellites over the years, their expected heterozygosity $H_{e}$ during the different time periods (see Figure 2) was determined. As expected, $H_{e}$ was smallest at the microsatellites directly adjacent to the $p f c r t$ locus and largest at the most distant microsatellites. During

Table 2 Prevalence of the local resistant haplotypes (100 kb) over the years

\begin{tabular}{lccc}
\hline & $\begin{array}{c}\mathbf{1 9 9 5 /} \\
\mathbf{9 6}\end{array}$ & $\mathbf{2 0 0 2}$ & $\begin{array}{c}\mathbf{2 0 0 5 -} \\
\mathbf{0 7}\end{array}$ \\
\hline no. of samples & 38 & 45 & 49 \\
\hline no. of samples with local haplotypes & 22 & 14 & 16 \\
$(\sim 100 \mathbf{~ k b})$ & $(58 \%)$ & $(31 \%)$ & $(33 \%)$ \\
\hline no. of samples with other haplotypes & 16 & 31 & 33 \\
$(\sim 100 \mathbf{~ k b})$ & $(42 \%)$ & $(69 \%)$ & $(67 \%)$ \\
\hline
\end{tabular}

all time periods $H_{e}$ increased with increasing distance from the $p f c r t$ locus. Furthermore, $H_{e}$ was higher at the centromeric microsatellites 9B12, 7A11 and PE14F compared to the telomeric microsatellites 3E7, B5M77 and PE14D, again suggesting a higher degree of genetic variability in the centromeric microsatellites. A trend towards increasing diversity over time was apparent for the two most centromeric microsatellites 7A11 and PE14F, however this was not statistically significant.

\section{Genetic diversity at the msp2 gene}

The analysis suggested that the genetic sweep on chromosome 7 persisted in the local parasite population despite the removal of CQ from the national treatment guidelines. To estimate the potential diversity in the Lambaréné parasite population, the expected heterozygosity at the locus coding for MSP2, a surface antigen with a presumably high diversity was also determined. The msp2 locus from 50 isolates of the years 2005- 07 was PCR amplified and the respective 3D7 (30) and FC27 (40) fragments were sequenced. A total of 48 different alleles were identified. The $H_{e}$ for the $m s p 2$ locus was calculated to be 0.98 . In comparison, the mean genomic $H_{e}$ of African P. falciparum parasites was assessed to be $0.76-0.8$ [28]. Of the microsatellites analysed in the 2005 - 07 population only the least conserved marker PE14F had a $H_{e}$ within that range $(0.76)$. Contrasting, the microsatellite B5M47 lying directly adjacent to the pfcrt locus had a $H_{e}$ of 0.11 (see Table $4)$. Taken together, this shows that the potential for genetic diversity is very high in this population and highlights the high degree of genetic conservation surrounding the mutant pfcrt gene on chromosome 7 .

\section{Discussion}

This is the first report analysing the effect of the withdrawal of CQ from the national treatment guidelines in 2003 on the prevalence of the mutant pfcrt allele in Lambaréné, Gabon. Prior to the change in national treatment policy the prevalence of the mutant $p f c r t$ allele $76 \mathrm{~T}$ had been $100 \%[13,14]$. However, even in the years 2005 - 07 the level of CQR was still as high as $97 \%$. Thus the decrease of CQR after discontinuance of CQ use observed in Gabon is much smaller compared to the development in Malawi where resistance decreased within eight years from $85 \%$ to $13 \%[3,4]$, as well as compared to other African countries such as Kenya [7] and Tanzania [6] where recent reports also documented a decrease in CQR from $95 \%$ to $60 \%$ and $94 \%$ to $70 \%$ respectively, after national CQ stops. What explanations can be invoked to explain the differences in the decline of CQR in these countries and Gabon?

The resurgence of CQS in Malawi, Kenya and Tanzania was observed after changes in national treatment 
Table 3 Prevalence of "inner" haplotype (40 kb) over the years

\begin{tabular}{lccc}
\hline no. of samples & $\mathbf{1 9 9 5 / 9 6}$ & $\mathbf{2 0 0 2}$ & $\mathbf{2 0 0 5 - 0 7}$ \\
\hline no. of samples with local "inner" haplotype ( 40 kb) & 43 & 47 & 55 \\
\hline no. of samples with other "inner" haplotypes ( 40 kb) & $34(79 \%)$ & $29(62 \%)$ & $32(58 \%)$ \\
\hline
\end{tabular}

guidelines from CQ to SP. In contrast, Gabon changed its national treatment guidelines from CQ to the combination of artesunate plus AQ. AQ is structurally related to CQ with both belonging to the group of 4-aminoquinolines. A recent report from Ghana analysed the prevalence of the mutant pfcrt allele after a change in national treatment guidelines from $C Q$ to artesunate and AQ. The authors observed a decrease in the prevalence of the mutant $p f c r t$ allele only in the northern part of the country, raising the question if this effect truly represented a national trend [29]. In contrast, a recent report from the Gabonese region of Franceville showed no change in the prevalence of the mutant $p f c r t$ allele after the change in national treatment guidelines [30]. Data from clinical trials and the 7G8xGB4 and HB3xDd2 genetic crosses clearly suggest that AQ strongly selects for the resistance conferring mutant pfcrt allele [31,32]. Analysis of progeny of the 7G8xGB4 genetic cross showed that the South American pfcrt haplotype SVMNT at the codons $72-76$ is associated with high level resistance against monodesethylamodiaquine, the active metabolite of AQ. Reports from Angola [33] and Tanzania [34] have shown the presence of the SVMNT $p f c r t$ haplotype following the use of artesunate and $A Q$ or the single use AQ, suggesting that AQ may select for this haplotype [35]. However, none of the 48 randomly sequenced mutant $p f c r t$ alelles from the years 2005 - 07 carried the SVMNT haplotpype in this analysis of the Lambaréné parasite population. In view of the high conservation of the chromosomal regions flanking the pfcrt gene even in 2005 - 07 this may simply reflect the continued selection of the most abundant $p f c r t$ haplotype CVIET. The introduction of the SVMNT haplotype may thus be influenced by the specific characteristics of local parasite populations. Taken together the data suggest that in Gabon the introduction of artesunate plus AQ may have selected for continued conservation of the mutant pfcrt locus.

Interestingly, a decrease in conservation of the local resistant chromosomal haplotypes was observed between $1995 / 96$ and 2002, prior to the removal of CQ from the national treatment guidelines. A similar phenomenon was observed in Kenya, where CQR started to decline prior to the official change in national treatment guidelines, possibly as a consequence of declining use due to decreased clinical effectiveness. Similarly, local treatment guidelines at the Albert Schweitzer Hospital were already changed in 1994 - from CQ to SP. A decrease in CQ pressure might thus have contributed to more genetic diversity surrounding the pfcrt locus, however the resurgence of CQS might have been precluded due to the continued use of CQ in the surrounding areas.

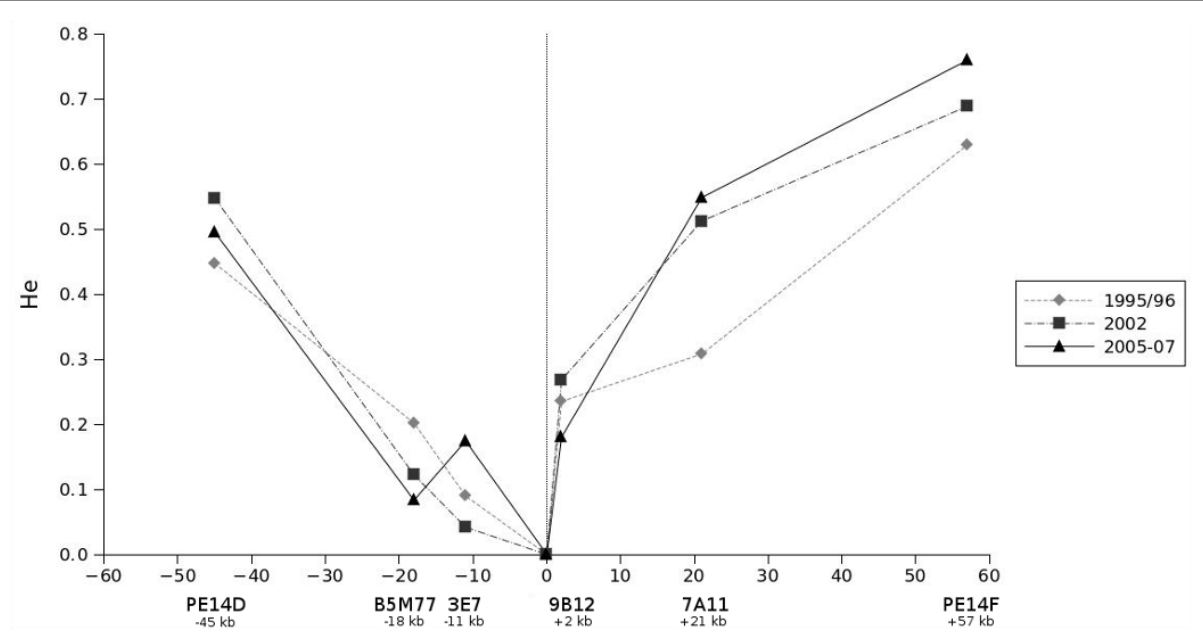

Figure 2 Expected heterozygosity around the pfcrt locus before and after the removal of chloroquine from national treatment guidelines. The expected heterozygosity $H_{e}$ is shown for each microsatellite in the resistant populations of the years 1995/96 (diamonds), 2002 (squares) and 2005-07 (triangles). On the $x$-axis the distance [kb] from the pfcrt locus is given. During all time periods $\mathrm{H}_{e}$ increased with increasing distance from the pfcrt locus but was higher at the centromeric microsatellites compared to the telomeric microsatellites. 
Table 4 Expected heterozygocity of B5M47 and PE14 F on chromosome 7 and expected heterotzygocity of the msp 2 locus on chromosome 2

\begin{tabular}{lccc}
\hline & B5M47 & PE14F & msp2 \\
\hline no. of samples & 54 & 69 & 50 \\
\hline no. of alleles & 3 & 9 & 48 \\
\hline $\mathrm{H}_{\mathrm{e}}$ & 0.11 & 0.76 & 0.98 \\
\hline
\end{tabular}

This is supported by an overall increase of different resistant haplotypes from 1995 to 2002 and by the decrease in prevalence of the most common "local resistant haplotypes".

What could be the mechanism that led to the reappearance of the wild type $p f c r t$ allele? None of seven point mutations in the pfcrt gene commonly found in CQR strains from Africa and Asia [11,36] were present in any of the genetically sensitive isolates, who thus appear to be genuine wild type $p f c r t$ alleles. This rules out the possibility that the sensitive isolates originated from a simple back mutation at the crucial position 76 of the pfcrt gene in former resistant parasites and is consistent with the results of others [3-5]. The implication of this finding is that the wild type $p f c r t$ allele must have been reintroduced into the current population through import from another geographic area, or by reexpansion of sensitive parasites that were under the detection level of previous investigations. However, given the persistently high prevalence of the resistant haplotypes the former possibility appears more likely. Chromosome 7 haplotype analysis of the three sensitive monoinfections showed no conservation of microsatellite alleles. Comparison to the local resistant population showed that one (isolate SP15) shared the two microsatellites PE14D and B5M77 at the most telomeric end of the analysed haplotype with the dominant local resistant haplotypes. To estimate the likelihood that the SP15 haplotype resulted from a cross of a local resistant and a sensitive isolate, the number of resistant parasites with the same PE14D and B5M77 alleles as SP15 was quantified. Of the 172 haplotypes analysed in the study, 107 carried the exactly same PE14D-7A11 microsatellite alleles as SP15, suggesting that SP15 might represent a genetic cross between a sensitive strain and a formerly resistant strain of the local population.

\section{Conclusions}

The data presented in this manuscript show that four years after the discontinuance of CQ as first-line therapy in Gabon the prevalence of the mutant pfcrt allele is still extremely high in the Lambaréné area. This finding stands in marked contrast to a more pronounced development towards CQS in some other African countries. One possible explanation may be an ongoing selection for the mutant pfcrt allele by AQ as part of an artemisinin-based combination therapy. It has repeatedly been proposed that CQ may return to clinical use in the future. The results of this investigation and the result of others [6,7] sound a note of caution and clearly show that this can only be contemplated in areas where the return of CQ sensitivity has clearly been demonstrated by molecular drug sensitivity monitoring.

\section{Additional material}

Additional file 1: Primers and conditions for microsatellite PCR amplification. Table of primer sequences and PCR cycling conditions employed for microsatellite analysis

\section{Acknowledgements}

Mrs. Andrea Weierich and Mrs. Velia Grummes were essential for the sequence analysis. This investigation was only possible because of the continued participation of the Lambaréné population in clinical investigations. Nicola Lehners was supported by the IZKF (Interdisziplinäres Zentrum für Klinische Forschung) of the University of Tübingen. Matthias Frank and Francine Ntoumi were supported by the CANTAM network. CANTAM is the Central Africa Network on Tuberculosis, HIV/AIDs and Malaria, jointly funded by the European and Developing Countries Clinical Trials Partnership (EDCTP) and the Netherlands-African partnership for capacity development and clinical interventions against poverty-related diseases (NACCAP).

\section{Author details}

${ }^{1}$ Institute of Tropical Medicine, University of Tübingen, Wilhelmstr.27, 72074 Tübingen, Germany. ${ }^{2}$ Medical Research Unit, Albert Schweitzer Hospital, Lambaréné, Gabon. ${ }^{3}$ Department of Medical Biometry, University of Tübingen, Germany. ${ }^{4}$ Infectious Diseases, Tropical Medicine and AIDS, Amsterdam Medical Center, University of Amsterdam, The Netherlands. ${ }^{5}$ Congolese Foundation for Medical Research/Faculty of Health Sciences, University Marien Ngouabi, Brazzaville, Congo. ${ }^{6}$ Département de Parasitologie, Université des Sciences de la Santé, Libreville, Gabon.

\section{Authors' contributions}

MF conceived the study and designed the experiments, NL carried out the molecular genetic studies on microsatellites and on the msp2 gene, PM carried out part of the msp2 analysis and extracted the DNA of part of the 1995/6 and 2002 samples, FN supervised part of the msp2 analysis, DUK and MDB conducted the Antigenic Diversity Study, JG, GMN, MG conducted the IPTi study and the SP Efficacy trial, GMN, CS, BL conducted the Ferroquine tolerance trial, KD performed the statistical analysis, PK coordinated the study and provided conceptual advice. All authors read and approved the final manuscript.

\section{Competing interests}

The authors declare that they have no competing interests.

Received: 6 August 2011 Accepted: 17 October 2011 Published: 17 October 2011

\section{References}

1. Trape JF: The public health impact of chloroquine resistance in Africa. Am J Trop Med Hyg 2001, 64:12-17.

2. Payne D: Spread of chloroquine resistance in Plasmodium falciparum. Parasitol Today 1987, 3:241-246. 
3. Kublin JG, Cortese JF, Njunju EM, Mukadam RA, Wirima JJ, Kazembe PN, Djimde AA, Kouriba B, Taylor TE, Plowe CV: Reemergence of chloroquinesensitive Plasmodium falciparum malaria after cessation of chloroquine use in Malawi. J Infect Dis 2003, 187:1870-1875.

4. Mita T, Kaneko A, Lum JK, Bwijo B, Takechi M, Zungu IL, Tsukahara T, Tanabe K, Kobayakawa T, Bjorkman A: Recovery of chloroquine sensitivity and low prevalence of the Plasmodium falciparum chloroquine resistance transporter gene mutation $\mathrm{K} 76 \mathrm{~T}$ following the discontinuance of chloroquine use in Malawi. Am J Trop Med Hyg 2003, 68:413-415.

5. Laufer MK, Thesing PC, Eddington ND, Masonga R, Dzinjalamala FK, Takala SL, Taylor TE, Plowe CV: Return of chloroquine antimalarial efficacy in Malawi. N Engl J Med 2006, 355:1959-1966.

6. Alifrangis $M$, Lusingu JP, Mmbando $B$, Dalgaard MB, Vestergaard LS, Ishengoma D, Khalil IF, Theander TG, Lemnge MM, Bygbjerg IC: Five-year surveillance of molecular markers of Plasmodium falciparum antimalarial drug resistance in Korogwe District, Tanzania: accumulation of the 581G mutation in the P. falciparum dihydropteroate synthase gene. Am J Trop Med Hyg 2009, 80:523-527.

7. Mwai L, Ochong E, Abdirahman A, Kiara SM, Ward S, Kokwaro G, Sasi P, Marsh K, Borrmann S, Mackinnon M, Nzila A: Chloroquine resistance before and after its withdrawal in Kenya. Malar J 2009, 8:106.

8. Fidock DA, Nomura T, Cooper RA, Su X, Talley AK, Wellems TE: Allelic modifications of the $\mathrm{cg} 2$ and $\mathrm{cg} 1 \mathrm{genes}$ do not alter the chloroquine response of drug-resistant Plasmodium falciparum. Mol Biochem Parasitol 2000, 110:1-10.

9. Sidhu $A B$, Verdier-Pinard D, Fidock DA: Chloroquine resistance in Plasmodium falciparum malaria parasites conferred by pfcrt mutations. Science 2002, 298:210-213.

10. Wellems TE, Walker-Jonah A, Panton LJ: Genetic mapping of the chloroquine-resistance locus on Plasmodium falciparum chromosome 7. Proc Natl Acad Sci USA 1991, 88:3382-3386.

11. Wootton JC, Feng X, Ferdig MT, Cooper RA, Mu J, Baruch DI, Magill AJ, Su XZ: Genetic diversity and chloroquine selective sweeps in Plasmodium falciparum. Nature 2002, 418:320-323.

12. Laufer MK, Takala-Harrison S, Dzinjalamala FK, Stine OC, Taylor TE, Plowe CV: Return of chloroquine-susceptible falciparum malaria in Malawi was a reexpansion of diverse susceptible parasites. J Infect Dis 2010, 202:801-808.

13. Binder RK, Borrmann S, Adegnika AA, Missinou MA, Kremsner PG, Kun JF: Polymorphisms in the parasite genes for pfcrt and pfmdr-1 as molecular markers for chloroquine resistance in Plasmodium falciparum in Lambarene, Gabon. Parasitol Res 2002, 88:475-476.

14. Borrmann S, Binder RK, Adegnika AA, Missinou MA, Issifou S, Ramharter M, Wernsdorfer WH, Kremsner PG: Reassessment of the resistance of Plasmodium falciparum to chloroquine in Gabon: implications for the validity of tests in vitro vs. in vivo. Trans R Soc Trop Med Hyg 2002, 96:660-663.

15. Mayengue PI, Kalmbach Y, Issifou S, Kremsner PG, Ntoumi F: No variation in the prevalence of point mutations in the Pfcrt and Pfmdr1 genes in isolates from Gabonese patients with uncomplicated or severe Plasmodium falciparum malaria. Parasitol Res 2007, 100:487-493.

16. Wildling E, Winkler S, Kremsner PG, Brandts $C$, Jenne L, Wernsdorfer WH: Malaria Epidemiology in the Province of Moyen-Ogoov, Gabon. Trop Med Parasitol 1995, 46:77-82.

17. Kun JF, Schmidt-Ott RJ, Lehman LG, Lell B, Luckner D, Greve B, Matousek P, Kremsner PG: Merozoite surface antigen 1 and 2 genotypes and rosetting of Plasmodium falciparum in severe and mild malaria in Lambarene, Gabon. Trans R Soc Trop Med Hyg 1998, 92:110-114.

18. Mayengue PI, Luty AJF, Rogier C, Baragatti M, Kremsner PG, Ntoumi F: The multiplicity of Plasmodium falciparum infections is associated with acquired immunity to asexual blood stage antigens. Microbes Infect 2009, 11:108-114.

19. Enderes C, Kombila D, Dal Bianco M, Dzikowski R, Kremsner P, Frank M: Var Gene Promoter Activation in Clonal Plasmodium falciparum Isolates Follows a Hierarchy and Suggests a Conserved Switching Program that Is Independent of Genetic Background. J Infect Dis 2011.

20. Rupp I, Sologub L, Williamson KC, Scheuermayer M, Reininger L, Doerig C, Eksi S, Kombila DU, Frank M, Pradel G: Malaria parasites form filamentous cell-to-cell connections during reproduction in the mosquito midgut. Cell Res 2011, 21:683-696.
21. Mombo-Ngoma G, Supan C, Dal Bianco MP, Missinou MA, Matsiegui PB, Ospina Salazar CL, Issifou S, Ter Minassian D, Ramharter M, Kombila M, Kremsner PG, Lell B: Phase I randomized dose-ascending placebocontrolled trials of ferroquine-a candidate anti-malarial drug-in adults with asymptomatic Plasmodium falciparum infection. Malar J 2011, 10:53.

22. Grobusch MP, Gabor JJ, Aponte JJ, Schwarz NG, Poetschke M, Doernemann J, Schuster K, Koester KB, Profanter K, Borchert LB, Kurth F, Pongratz $P$, Issifou $S$, Lell $B$, Kremsner PG: No rebound of morbidity following intermittent preventive sulfadoxine-pyrimethamine treatment of malaria in infants in Gabon. J Infect Dis 2009, 200:1658-1661.

23. Grobusch MP, Lell B, Schwarz NG, Gabor J, Dornemann J, Potschke M, Oyakhirome S, Kiessling GC, Necek M, Langin MU, Klouwenberg PK, Klopfer A, Naumann B, Altun H, Agnandji ST, Goesch J, Decker M, Salazar CL, Supan C, Kombila DU, Borchert L, Koster KB, Pongratz P, Adegnika AA, Glasenapp I, Issifou S, Kremsner PG: Intermittent preventive treatment against malaria in infants in Gabon-a randomized, doubleblind, placebo-controlled trial. J Infect Dis 2007, 196:1595-1602.

24. Mombo-Ngoma G, Oyakhirome S, Ord R, Gabor JJ, Greutelaers KC, Profanter K, Greutelaers B, Kurth F, Lell B, Kun JFJ, Issifou S, Roper C, Kremsner PG, Grobusch MP: High prevalence of dhfr triple mutant and correlation with high rates of sulphadoxine-pyrimethamine treatment failures in vivo in Gabonese children. Malar J 2011, 10.

25. Djimde A, Doumbo OK, Cortese JF, Kayentao K, Doumbo S, Diourte Y, Dicko A, Su XZ, Nomura T, Fidock DA, Wellems TE, Plowe CV, Coulibaly D: A molecular marker for chloroquine-resistant falciparum malaria. New Engl J Med 2001, 344:257-263.

26. Su XZ, Ferdig MT, Huang YM, Huynh CQ, Liu A, You JT, Wootton JC, Wellems TE: A genetic map and recombination parameters of the human malaria parasite Plasmodium falciparum. Science 1999, 286:1351-1353.

27. Ntoumi F, Ngoundou-Landji J, Lekoulou F, Luty A, Deloron P, Ringwald P: Site-based study on polymorphism of Plasmodium falciparum MSP-1 and MSP-2 genes in isolates from two villages in Central Africa. Parassitologia 2000, 42:197-203.

28. Anderson TJ, Haubold B, Williams JT, Estrada-Franco JG, Richardson L, Mollinedo R, Bockarie M, Mokili J, Mharakurwa S, French N, Whitworth J, Velez ID, Brockman AH, Nosten F, Ferreira MU, Day KP: Microsatellite markers reveal a spectrum of population structures in the malaria parasite Plasmodium falciparum. Mol Biol Evol 2000, 17:1467-1482.

29. Alam MT, de Souza DK, Vinayak S, Griffing SM, Poe AC, Duah NO, Ghansah A, Asamoa K, Slutsker L, Wilson MD, Barnwell JW, Udhayakumar V, Koram KA: Selective Sweeps and Genetic Lineages of Plasmodium falciparum Drug -Resistant Alleles in Ghana. J Infect Dis 2011, 203:220-227.

30. Lekana-Douki JB, Boutamba SDD, Zatra R, Edou SEZ, Ekomy H, Bisvigou U, Toure-Ndouo FS: Increased prevalence of the Plasmodium falciparum Pfmdr1 $86 \mathrm{~N}$ genotype among field isolates from Franceville, Gabon after replacement of chloroquine by artemether-lumefantrine and artesunatemefloquine. Infect Genet Evol 2011, 11:512-517.

31. Djimde AA, Fofana B, Sagara I, Sidibe B, Toure S, Dembele D, Dama S, Ouologuem D, Dicko A, Doumbo OK: Efficacy, safety, and selection of molecular markers of drug resistance by two ACTs in Mali. Am J Trop Med Hyg 2008, 78:455-461.

32. Sa JM, Twu O, Hayton K, Reyes S, Fay MP, Ringwald P, Wellems TE: Geographic patterns of Plasmodium falciparum drug resistance distinguished by differential responses to amodiaquine and chloroquine. Proc Natl Acad Sci USA 2009, 106:18883-18889.

33. Gama BE, Pereira-Carvalho GA, Lutucuta Kosi FJ, Almeida de Oliveira NK, Fortes F, Rosenthal PJ, Daniel-Ribeiro CT, de Fatima Ferreira-da-Cruz: Plasmodium falciparum isolates from Angola show the StctVMNT haplotype in the pfort gene. Malar J 2010, 9:174.

34. Alifrangis $M$, Dalgaard MB, Lusingu JP, Vestergaard LS, Staalsoe T, Jensen AT, Enevold A, Ronn AM, Khalil IF, Warhurst DC, Lemnge MM, Theander TG, Bygbjerg IC: Occurrence of the Southeast Asian/South American SVMNT haplotype of the chloroquine-resistance transporter gene in Plasmodium falciparum in Tanzania. J Infect Dis 2006, 193:1738-1741

35. Sa JM, Twu O: Protecting the malaria drug arsenal: halting the rise and spread of amodiaquine resistance by monitoring the PfCRT SVMNT type. Malar J 2010, 9:374.

36. Fidock DA, Nomura T, Talley AK, Cooper RA, Dzekunov SM, Ferdig MT, Ursos LM, Sidhu AB, Naude B, Deitsch KW, Su XZ, Wootton JC, Roepe PD, 
Wellems TE: Mutations in the P. falciparum digestive vacuole transmembrane protein PfCRT and evidence for their role in chloroquine resistance. Mol Cell 2000, 6:861-871.

doi:10.1186/1475-2875-10-304

Cite this article as: Frank et al: A thirteen-year analysis of Plasmodium falciparum populations reveals high conservation of the mutant pfcrt haplotype despite the withdrawal of chloroquine from national treatment guidelines in Gabon. Malaria Journal 2011 10:304.

Submit your next manuscript to BioMed Central and take full advantage of:

- Convenient online submission

- Thorough peer review

- No space constraints or color figure charges

- Immediate publication on acceptance

- Inclusion in PubMed, CAS, Scopus and Google Scholar

- Research which is freely available for redistribution

Submit your manuscript at www.biomedcentral.com/submit
C) Biomed Central 